\title{
Article
}

\section{How to Successfully Manage Entrenched Conflict in Mediation}

\author{
Sheila Gooderham*
}

\section{Introduction}

A wise mediation mentor once said to me years ago, "If the mediator is working harder than the clients to resolve the dispute, then something is very wrong". All mediators are likely to face particularly challenging mediations from time to time during the course of their career. Of these more complex cases, a sizeable minority will be entrenched disputes. These are the conflicts which are very difficult to manage, appear intractable and leave a mediator feeling frustrated and unable to make normal progress. Entrenched conflict in the mediation context occurs when participants assert that they wish to engage in mediation and instruct a mediator, but then one or both disputants demonstrate no genuine willingness to proactively engage in the mediation process. They display no willingness to compromise in order to reach a settlement, despite the mediator's best efforts.

In entrenched conflict cases, mediation participants display a contradictory approach. They fail to take responsibility for their part in mediation and do not engage constructively in negotiations, whilst asserting a justificatory narrative for their behaviour. Usually they blame the other disputant, make excuses based on extraneous factors or even assert that the mediator is to blame for the lack of progress in mediation. In many entrenched conflict cases, there is no genuine commitment to negotiation at all on the part of the entrenched disputant. They are simply keen to present their case with an

Sheila Gooderham is a writer, lawyer-mediator and director of The Mediation Specialists. expectation that everyone else will fall into line with their demands. When entrenched conflict manifests, mediation is often being used as a forum for psychological game playing. Entrenched disputants tend to have a 'win at all costs' perspective. In some entrenched cases, mediation is simply being used as a tactic, with a view to fighting the case in court. In such circumstances, the entrenched disputant may simply see mediation as a means of eliciting further information about their opponent's case, so as to benefit the entrenched disputant in subsequent court proceedings.

\section{How Common Is Entrenched Conflict in the Mediation Process?}

Entrenched conflict can manifest in all mediation forums whether commercial, workplace or in the private/family law sector. Generally, the more emotive the subject matter of the dispute, the more likely entrenched conflict is to occur. My personal experience as a mediator reflects my general research into success rates of mediation nationally and internationally over the years. It is clear that mediation has a fairly consistent general success rate of $70 \%$, regardless of the quality or variance of the mediation process offered to disputants. This reflects the fact that over two thirds of those who attend mediation are genuinely committed to reaching a settlement through the mediation process. However, $30 \%$ of all disputes, whether commercial, civil, workplace or family, are harder to settle and some may always be impossible to do so by means of mediation. It is within the $30 \%$ failure rate that the entrenched con- 
flict cases lurk, however good the mediator may be. It is certainly possible to increase this $70 \%$ general settlement success rate to between 80 and $90 \%$ with the right amount of leverage and advanced mediation techniques. The chances of achieving success in mediation have universally improved over the years due to societal leverage. Mediation has become more widely known and mainstream as a form of dispute resolution. Increased societal expectation, and social pressure to settle disputes by means other than court-based litigation, helps overcome resistance to mediation. As the cost and time benefits of mediation versus litigation are more widely promoted, this helps to encourage prospective clients to mediate. The culture of litigating disputes has been gradually changing. The court is now seen as the last resort in many countries rather than the first resort. This shift in emphasis away from the adversarial system means that it is more difficult for highly litigious and adversarial disputants, who are hostile to the concept of concession and negotiation, to go straight to court. Indeed, in many countries these clients have little alternative but to go to mediation first or risk judicial rebuke, or even costs orders, if they fail to try to mediate first.

Courts are, generally, now more likely to penalise litigants who do not engage constructively in mediation and, subsequently in the court process, this can act as a disincentive to litigate for many disputants. For others, regardless of their outlook, mediation may be the only real option due to the prohibitive costs of litigation. However, for some high conflict clients, mediation may actually be welcomed. They may see it as a 'soft option' and incorrectly believe that their mediator will be easier to influence than a judge. High emphasis, and even conflict, over the choice of mediator at the outset of the case may be indicative that one or both participants believe that one mediator may be more favourable to them than another. However, given that the vast majority of mediators have the necessary skills to address attempts to manipulate the mediation process, entrenched disputants are likely to be disappointed by the robustness of the mediation process and, therefore, also antagonistic towards mediation and the mediator, once the mediation process actually begins and the extent of the neutrality of the mediator is understood.

Mediation, particularly where the mediator is highly experienced and trained in advanced mediation skills, has the capacity to effectively manage entrenched conflict and increase mediation settlement success rates, in spite of client resistance. This seems to be borne out by the UK audit of civil and commercial mediation in 2018. The Centre for Effective Dispute Resolution (CEDR) released its most recent set of civil mediation results by way of its eighth biennial 'Mediation Audit', in conjunction with the UK Civil Mediation Council. The 2018 audit received 336 responses from $\mathrm{UK}$-based civil and commercial mediators. Whilst such reviews and audits are, by their nature, limited and based on a small sample of participants, the audit's findings are interesting in that they provide an indicator of trends. The audit fig- ures show that the net is tightening on those who are more reluctant mediation participants, as dispute culture changes and mediation consolidates its place as a mainstream, and universally acceptable and effective, form of dispute resolution.

CEDR's 2018 civil and commercial mediation audit confirmed the increasing popularity of mediation as an effective dispute resolution method over the previous two years. In the last 12 months prior to the audit, CEDR reported that 12,000 commercial mediations generated an estimated claim value of $£ 11.5$ billion. The largest increase in mediation cases was those that took place through organisational mediation processes, such as the National Health Service's 'Resolution' scheme, and the County Court pilot, and the Court of Appeal schemes, where mediation uptake grew by $45 \%$ since 2016. However, individual referred cases to mediation, which occur on an ad hoc basis and are not funnelled to mediation via a particular scheme, only increased by $9 \%$ since 2016, so there is clearly more work to be done in encouraging those disputants outside the remit of mediation schemes to enter mediation.

The CEDR 2018 Mediation Audit indicated that the success rate for commercial mediation remained high and indeed continued to increase with an overall settlement rate of $89 \%$ ( $86 \%$ in 2016$)$, of which $74 \%$ achieved settlement on the day of mediation and 15\% shortly after mediation. The Mediation Audit 2018 asked mediators for their views on the performance of those lawyers and clients that attended mediations. The mediators reported that $63 \%$ of lawyers and clients performed well, or quite well, in mediation, $23 \%$ of clients were rated as performing adequately and 14\% of clients were rated as performing less than adequately (with 5\% of lawyers and clients rated as having been very poor). It is this latter small group, probably accounting for between 10 and 15\% of disputants, which are likely to be identified as engaging in entrenched conflict behaviour and are far less likely to achieve a settlement through mediation. However, some basic strategies can improve the chances of a successful outcome in even the most challenging mediation cases.

\section{A Few Basic Strategies for the Management of Entrenched Conflict}

In all disputes, the mediator's role is to facilitate negotiations and, in so doing, ensure that the mediation process is not manipulated and boundaries of conduct are maintained at all times. This is particularly important in entrenched conflict cases where boundary challenges are inevitable. What can the mediator do to improve the chances of achieving a mediated settlement in entrenched conflict cases? 


\subsection{Maintain No Attachment to Outcome}

It is important for a mediator not to be fixated with settling a case through mediation. Being unable to resolve a dispute through mediation is not a failure of mediation. As a neutral facilitator, it is important to remain mindful that mediation is not suitable in every case. It is important to identify participants who are not prepared to genuinely engage in constructive negotiations at the earliest opportunity and refer them on to a more suitable dispute resolution process for their case, if necessary. Sometimes, when a mediator simply expresses concern to both clients that mediation may not be the most appropriate forum, and if it proves not to be, then the mediator will stop the process. This assertion of discretion is sufficient to focus the minds of clients and incentivise cooperation within mediation.

Early warning signs of client non-commitment to mediation can include lack of preparation of appropriate mediation document bundles, or late and incomplete submission of documents. It is important that the mediator makes it clear that high standards of disclosure are expected and that timetables for disclosure must be adhered to. In such cases, where suitability of client mindset may be of issue, it can be fairer to both participants for the mediator to refuse to mediate until disclosure is complete and charge extra fees to the obstructive disputant who causes a delay in the process. When a client fails to comply with prescribed requirements, it jeopardises the quality of the mediation process. It is the mediator's responsibility as facilitator to ensure that the mediation process is not sub-standard, due to client sabotage. It is better to cancel mediation than to engage in a poor process. Ensuring that all parties are aware from the outset that failure to adhere to the mediation timetable will incur additional mediation fee sanctions and that delayed disclosure will not be tolerated can be a further incentive to full compliance and respect for the mediation process. This ensures that, when mediation does commence, all necessary information is at hand to resolve the dispute.

Informing clients from the outset that it is within the mediator's remit to call a halt to mediation, if necessary, can avoid game playing from the start and can be included in the mediation contract, with attention drawn to these terms. Clients have an expectation that mediation has no teeth, unlike court intervention, where there is the power to make costs orders for non-compliance with court directions. They assume, because they are paying for the service, that there will be no such repercussions should they breach mediation protocols. It is up to the mediator to value their time and to protect the mediation process from the start, using strict requirements to create a tone of respect and high expectation. A willingness on the part of the mediator to be firm and to put the value on the process means the mediator has no attachment to outcome in terms of the clients' attempts to manipulate or undermine the mediation process. Being prepared to let go of a case, without attachment at any stage in the process, is vital when handling entrenched disputants. The strength of resolve of the mediator to maintain a fair process from the outset pays dividends all round and ensures only those with the appropriate mindset for mediation engage in it.

Over the years, I found much of my referred mediation work came from lawyers who trusted that I would not take on a case or proceed with mediation if I identified that one or both participants were attempting to manipulate the mediation process, or if I felt that another alternative dispute resolution process, or the court system, would be a more suitable forum. Mediation is not a 'one size fits all' process and, whilst the range of disputes which are suitable for mediation is extremely high, it is not universal. However, whilst mediation cannot resolve all disputes, an experienced mediator can do a lot to unlock impasse in the more entrenched cases that they deem fit to attempt to facilitate. At the very least, mediation can narrow the issues of dispute and, with careful management, much can be done to improve prospects of success, even in cases which initially appear to be high conflict. So, what can be done to unlock entrenched conflict when the mediator decides to take on a tough case?

\subsection{Preparation, Preparation and More Preparation}

For those of us familiar with the mediation process, it is easy to forget that mediation is still a new and unfamiliar concept for many clients, regardless of their background. Mediation is not easy for clients to understand, when compared with the 'old regime' of court-based litigation, which has operated in society for hundreds of years. How can the client trust that the mediator will be neutral, for instance, or robust, if the mediator does not determine the case? Mediation is more casual. No orders are imposed. What if the mediator is younger than the disputants, a different gender or comes from an entirely different ethnic and socio-economic background from the clients? The unfamiliarity of the process makes it feel unsafe to those who are fearful and entrenched. Mediation can seem daunting and a somewhat amorphous concept to understand, let alone participate in, for many clients.

Explaining mediation carefully is essential. I found, over the years, that spending a significant period of premediation time connecting with and listening to new clients made all the difference. I found that educating clients as to my role, and what to expect in the mediation, was invaluable. My one-to-one meetings with clients established trust in me, and trust in the process. Understanding a client's perspective, and answering any questions that they might have in a more relaxed one-to-one pre-mediation meeting, created the opportunity to allay client fears and concerns, as well as giving me insight into the nature and personalities of the clients that I was about to mediate, and how best to manage the mediation in the light of that. Shifting perspectives from one of distrust, fear, concern and anxiety, to one of trust and familiarity, almost always made a real difference to whether the outcome of mediation was successful, 
because the willingness of the disputants to participate and engage constructively in mediation is vital.

\subsection{Early Identification of High Conflict Personalities and Entrenched Conflict Indicators}

So, what are the general characteristics of entrenched, high conflict, mediation disputes? Entrenched conflict may occur when one or more disputants display one or more of the following characteristics within the mediation process:

1. Inability to work collaboratively. (Disputants only see teamwork as being in their interests, to the extent that they can use it as a means to manipulate others.)

2. Withholding or distortion of information.

3. Attacking others who attempt to manage or evaluate them.

4. Acting in a highly competitive manner.

5. Attempt to disrupt or manipulate the mediation process.

6. Demands for special treatment by way of asserting entitlement to higher status than others and treating others as pawns in the disputant's drama.

7. Inability to share. (Disputants will not share relevant information unless they have an ulterior motive.)

8. Inability to tell the truth. (Disputants cross back and forth between lies and honesty easily. Their dishonest statements are often interwoven with a thread of truth. Thus, if questioned, they will indignantly point out the true elements in their defence and ignore the false statements.)

9. Inability to be modest, possessing an arrogant and inflated attitude.

10. Inability to accept blame/take responsibility for actions. (They not only blame others, but create evidence that others are to blame. They seize an opportunity to bring harm to others' status, careers or professional standing through active, instrumental, aggression.)

11. Inability to act predictably.

12. Inability to act calmly.

13. Inability to act without some form of aggression (be that bullying, intimidation, passive or overt aggression, manipulation or coercion).

14. Inability to respect boundaries (for instance, time limits, punctuality, requests for information or the rules of the mediation process).

Understanding that fear is at the root of all these behaviours can be helpful for the mediation process.

\subsection{Understanding That Fear Is Behind Entrenched Conflict}

Behind every entrenched conflict is fear. The underlying fear of all human beings is a fear of losing. At its core, this fear is primaeval. The human brain is designed to protect us from loss of life. There are survival triggers that can create rigid, rather than diverse and flexible, thinking when the brain detects that we are under threat. This fear of loss of life is a ghost from our past history when we inhabited the world in loin cloths, wielded clubs and were lunch for wild animals. In most human disputes in the modern world, we are not in mortal danger. The threat is emotional rather than physical. However, whilst the stakes for survival aren't so high, our subconscious fears may still exist and can manifest as a fear of losing out, of losing in a business deal, losing face with our boss or in an emotional struggle. For some, they associate losing with being at the bottom of the pile. Subconsciously, they see losing in a dispute with another human being as a form of social annihilation and this creates fear. Fear occurs when we see the world around us, and those we interact with, as fundamentally hostile and threatening to our well-being. Therefore, entrenched conflict is a fear-based approach and perspective on life.

The existence of fear may not be something which we immediately associate with a commercial dispute. However, fear on some level is the underlying current which creates a strong attachment to a certain outcome and, in particular, 'out gunning' the opposition, or of losing face as a consequence of 'losing' in the negotiations. Fear may be concealed under a veneer of posturing, obstruction and aggression, whether that appears as a passive or as an actively destructive approach to mediation. Either are indicators of a rigid and inflexible mindset, resistant to moving forward other than on the disputant's set terms. Entrenched conflict is an attempt by one or both participants to control their external environment so as to make it feel safe for them.

Fear, when it is acute in the mediation process, manifests as 'high conflict' and it brings out the 'fight' or 'flight' response in clients. When, as mediators, we understand the mechanics, and in particular the brain science, behind fear we not only understand our clients better, but also ourselves and our reactions to intransigence in the mediation process. It is important to become conscious of the stress we experience when engaged in the management of high conflict. Fear is contagious and when we react with frustration, however well concealed, it often can have an impact upon negotiations. With real understanding and education around the dynamics of fear and how it manifests, it is possible to see fear as a universal problem rather than something specific to the challenge at hand. In this way, fear becomes less personal, more a human problem to be solved than a personal reflection on the qualities of the mediator.

In private mediations, I found it helpful to explain the dynamics of fear to clients in one-to-one sessions before joint mediation began. The knowledge and insights that I provided about how the brain responds to perceived threat revolutionised my clients' experience of the mediation process, not just in terms of outcomes, but in terms of the transferable skills that my clients learned and took away with them, beyond the mediation process, to use again in future challenging circumstances. Certainly, an understanding of fear management increases the chances of successful mediation outcomes. In 
more entrenched disputes it enabled clients to gain selfinsight and step back and reflect on their experience and how and why they are responding to the stress of mediation in a certain way.

My interest in how and why we all behave the way we do grew during the course of my mediation career. Over time I felt compelled to research fear and to educate myself on the motivators behind human conflicts. 'How to Handle Fear in Daily Life and Every Crisis' is the working title of the book I am writing, which addresses the neuroscience behind fear and how to manage fear, when it manifests within us, and also in others. When we understand the dynamics of fear and the fear-based belief systems that create intransigence, this enhanced mediation knowledge can unlock impasse. When we change and have insight, our mediation practice changes for the better too.

Often, all it takes to break a deadlock is for a mediator to understand the subconscious driving force behind obstructive behaviour. Simply understanding a disputant's fear then provides the opportunity to give the support necessary to address the fear, within the mediation context. It allows the disputant to feel understood and that shift in mediator-client dynamics can lead to more cooperation and movement from the disputant within negotiations. Also, once the disputant's fear is known to the mediator, even if not discussed directly with the client, the mediator is often then able to identify a suitable avenue for overcoming that fear.

\section{Consider Adjusting the Standard Mediation Model in Entrenched Conflict Cases}

Ultimately, the responsibility for having a proactive and constructive approach to negotiations lies with the disputants, but there are certain adjustments that a mediator can make to the mediation process which may be helpful in high conflict and entrenched disputes.

\subsection{Are Joint Meetings Really Necessary?}

The CEDR 2018 Mediation Audit recorded other interesting indicators which may give a hint at some of the reasons for the continued rise in success of commercial mediation outcomes. A striking $25 \%$ of commercial mediator comments, in response to the audit, indicated that clients were increasingly resistant to joint meetings at the start of the mediation day. However, a number of mediators reported seeing an increase in joint meetings between lawyers and/or clients later on in the course of the mediation. Could this be linked to the increase in the success rate of mediation?

There is no doubt in my mind why a reduction in joint meetings across the board and, regardless of the nature of mediation, may be helpful. In my professional experience as a mediator, eliminating joint meetings in entrenched cases until further on into the process was helpful. In acute cases, settlement was achieved without any joint meetings at all. By avoiding joint meetings, more reluctant (and thus fearful) mediation participants can be shielded from emotive triggers that may sabotage their ability to think clearly and engage constructively in mediation. When such clients remain 'safely' ensconced in their individual meeting rooms on mediation day, without the imminent prospect of a joint meeting, their 'fear' levels and emotional tension is lowered. This means they face less distraction and therefore their focus can be channelled and maintained on achieving a negotiated outcome. This makes life easier for the mediator who can focus on the parameters for settlement rather than on managing client emotions. In my experience, in entrenched conflict cases, there is no reason why there should be any joint meetings at all. Indeed, technology means that they need not even be in the same building.

As mediators, being flexible as to the way we approach the mediation process, whilst maintaining the core principles of neutrality and confidentiality of the process, is vital. When facing entrenched conflict, flexibility as to the structure of the process can go a long way. When we take into account the personalities and organisational culture of those that we are mediating, and any indicators of entrenched conflict that may come to light, then adjustments in process can make a huge difference to outcome. Those disputants who are most reluctant to mediate and most resistant to negotiation and compromise invariably have serious concerns as to the efficacy of the mediation process and the outcome of negotiations. Their fears are easily triggered. The prospect of joint meetings can act as a deterrent to mediating at all particularly in emotive cases. In many of my high conflict cases, I offered shuttle mediation so that the participants never had to meet at all. In extreme cases where there had been some form of domestic abuse, I even mediated settlements by way of meetings on separate days so that the stress of knowing that an opponent was in the same building was removed. This worked very well.

It is important to remember that, even if the subject matter of mediation seems commercial rather than personal, those engaged in the process of negotiation are human beings with their own fears and concerns about the outcome of the process and their own careers potentially on the line. Being a disputant is a stressful experience. There may be unseen corporate dynamics at play and an agenda or culture that is impacting on the prospect of success. In all my mediations, from the commercial to the family-based disputes, I found pre-mediation one-to-one meetings helpful, but also accommodating the need for separate meetings and being flexible as to process, was vital. Therefore, it is important that mediators adapt the mediation process to meet the needs of those that are being mediated, so that the process is as comfortable as possible and fear, uncertainty and tension is kept to a minimum. 


\subsection{Consider Use of Lawyers or Other} Supportive Third Parties in the Process

The 2018 audit also showed an increase in the use of third parties within the mediation process. In entrenched and challenging mediation cases, the involvement of third parties, such as the disputant's lawyer, can often make a positive difference, albeit it is important to check out first whether the lawyer is supportive or hostile to the mediation process. Most lawyers are an invaluable support to clients undertaking mediation and are positive about mediation. Stressbased emotions can make usually clear thinking clients less able to think clearly. As a result, having a clearheaded objective professional lawyer present can be very helpful. The client's lawyer is more likely to be pragmatic and objective and give sound advice to the client in terms of reaching a compromise. For the client to pay extra legal fees to have legal support present is usually money well spent, if it avoids the breakdown of mediation.

Whilst disputants often fare better in terms of lowered stress levels, and thus emotional capacity to settle, when accompanied by their professional support, it is also important for mediators to operate in a way that works best for them, and not be inclined to adjust the process to suit the lawyers. So, whilst professionals accompanying clients may work in many cases, it may not always be suitable if the mediator does not feel that the involvement of specific professionals will be constructive or enhance the process. After all, the process must be driven by the mediator and what works for that individual. Whilst accommodation and adaptability may create opportunities for some mediation clients who would not otherwise be able to mediate due to their fears, ultimately, as mediators, we must craft a process that works for us in order for the process to be sustainable and most effective.

\section{Acknowledging the Challenges of Managing Entrenched Conflict}

As mediators, it is the quality and energy we bring to mediation that counts. It is our capacity to understand, adapt and yet remain strong and confident that holds the mediation process together. As my mentor said, if as mediators, we are working too hard to create the perfect process for our clients, such that our needs and the integrity of the process become lost, then something is wrong. If we are to stay the course as mediators and remain fit, well, committed and energised so that we can cope with the inevitable challenges of mediating more emotionally complex cases, we must be prepared to draw the line swiftly under those cases more suited to an imposed, rather than a collaborative and negotiated, outcome. This avoids mediator burnout.
Provided a mediator is doing their best, ultimately the outcome of mediation is rarely a reflection on the mediator's performance or skills, but indicative of human nature. It takes all sorts of people to make a world and all sorts of dispute processes. Some cases are always more difficult, or even impossible, to settle because some clients are always more fearful, and thus obstructive, than others. All of life is reflected in the mediation process. It is a microcosm of human responses and the trickier end of human relationships. It is a forum that reflects the whole spectrum of human nature and emotions in the face of adversity, including fear in all its entrenched forms. As independent neutrals, mediators are facilitators and observers. We have a ringside view of human nature and it is a privilege to do our job. We are the guardians of our mediation process and its values of fairness, responsibility, integrity and respect.

Over my years as a lawyer and mediator, I have come to believe that each mediation case has a successful outcome, regardless of whether an overall settlement is reached. Sometimes one participant feels vindicated and validated by the process, sometimes both. Sometimes issues are narrowed; sometimes alternative perspectives are finally understood and respected. Sometimes previously covert adverse behaviour is exposed for the first time. On other occasions, a sense of peace and satisfaction and agency is achieved by all participants when they reach a settlement and mediation is truly transformational. In many cases, 'The law of equal disgruntlement' applies. Even in cases which have to be referred on to the court or arbitration, there is always learning to be had. Regardless of the outcome, all mediations provide the mediator with a further invaluable layer of experience that will enhance the next mediation. Without my more entrenched and challenging mediation cases, I would never have felt compelled to study the neuroscience of fear, and I would never have written my book or this article. 